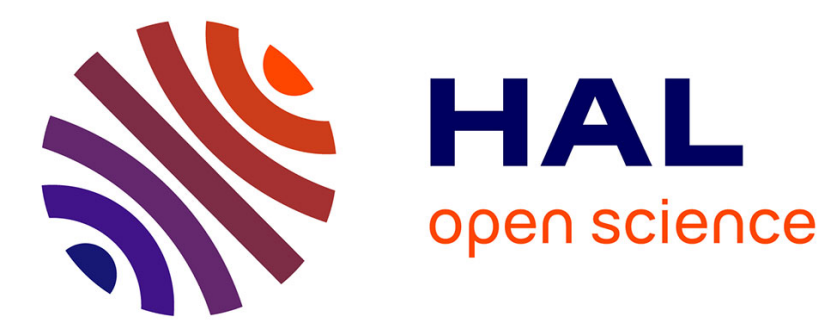

\title{
Development of 3D PUFEM with linear tetrahedral elements for the simulation of acoustic waves in enclosed cavities
}

\author{
M. Yang, E. Perrey-Debain, Benoit Nennig, J.-D. Chazot
}

\section{- To cite this version:}

M. Yang, E. Perrey-Debain, Benoit Nennig, J.-D. Chazot. Development of 3D PUFEM with linear tetrahedral elements for the simulation of acoustic waves in enclosed cavities. Computer Methods in Applied Mechanics and Engineering, 2018, 335, pp.403-418. 10.1016/j.cma.2018.03.002 . hal01743866

\section{HAL Id: hal-01743866 \\ https://hal.science/hal-01743866}

Submitted on 17 Apr 2018

HAL is a multi-disciplinary open access archive for the deposit and dissemination of scientific research documents, whether they are published or not. The documents may come from teaching and research institutions in France or abroad, or from public or private research centers.
L'archive ouverte pluridisciplinaire HAL, est destinée au dépôt et à la diffusion de documents scientifiques de niveau recherche, publiés ou non, émanant des établissements d'enseignement et de recherche français ou étrangers, des laboratoires publics ou privés. 


\title{
Development of 3D PUFEM with linear tetrahedral elements for the simulation of acoustic waves in enclosed cavities
}

\author{
M. Yang*, E. Perrey-Debain*, B. Nennig ${ }^{+}$, J.-D. Chazot* \\ * Laboratoire Roberval - FRE CNRS 2012 \\ Sorbonne Universités, Université de Technologie de Compiègne, \\ CS 60319, 60203 Compiègne cedex, France \\ + Institut Supérieur de Mécanique de Paris (SUPMECA), Laboratoire Quartz EA 7393, \\ 3 rue Fernand Hainaut, 93407 Saint-Ouen, France.
}

\begin{abstract}
This work is concerned with the numerical simulation of sound pressure field in three-dimensional cavities in which absorbing materials are present. Standard techniques such as the Finite Element Method are known to be extremely demanding computationally when the frequency increases and thus limited to low frequency applications. To alleviate these difficulties, an alternative formulation based on the Partition of Unity Finite Element Method is proposed. The method involves enriching the approximation finite element space by expanding the acoustic pressure in a set of plane waves propagating in various directions over the unit sphere. Particular attention is devoted to the fast and accurate computation of highly oscillatory integrals which is required by the method. Convergence studies show that these wave finite elements allow to capture accurately the wave field with a number of degrees of freedom that only grows quadratically with the frequency yielding drastic data reduction compared to classical FEM. Results of practical interest are shown for the case of a sound source placed in a reverberation room with absorbing materials.
\end{abstract}

This archived file is not the final published version of "M. Yang , E. Perrey-Debain , B. Nennig , J.-D. Chazot, Development of 3D PUFEM with linear tetrahedra elements for the simulation of acoustic waves in enclosed cavities. Comput. Meth. Appl. Mech. Eng. 335, 403-418, (2018)". Contact the publisher for reprint or permission to use the material in any form. The definitive publisher-authenticated version is available online at https://doi.org/10.1016/j.cma.2018.03.002.

Keywords: Partition of unity finite element method, Helmholtz equation, highly oscillatory integrals.

\section{Introduction}

Traditional Finite Element Method (FEM) is not efficient enough to solve medium and high frequency acoustic waves because of excessive demands involving heavy computational cost. This results from the thumb rule which indicates that at least 10 nodal points per wavelength are required. Moreover FEM is known to suffer from the so-called pollution error which requires a fine mesh discretization, especially at high frequency, in order to maintain acceptable accuracy.

New deterministic prediction techniques have been developed in the recent years to overcome this limitation. Mainly developed for domain-based methods, one direction of particular interest to us is the reduction of the complexity by expanding the dynamic field variable with a set of oscillatory wave functions which are the analytical solutions to the governing equation of the problem. These techniques include the Partition of Unity Finite Element Method (PUFEM) [1, the Ultra-Weak formulation [2, Wave-Based Methods [3], the Discontinuous Galerkin Method [4] and the Variational Theory of Complex Rays [5, 6]. All of these methods can offer a drastic reduction in degrees of freedom compared with conventional FEM. Among them, PUFEM offers the advantage of being very similar to the FEM, can be easily adapted to any FEM mesh and has 
been successfully used to solve acoustic wave scattering [7, 8, flow acoustic [9] and other wave propagation problems including porous and poroelastic materials [10, 11].

Although these prediction techniques allow a huge reduction in the number of degrees of freedom, the computation of element matrices requires the integration of highly oscillatory functions leading to expensive computational costs due to the need of a too large number of integration points. These limitations can be prohibitive especially for 3D simulations. Therefore, several new integration schemes were developed to improve competitiveness of the PUFEM and in this regard we can cite the earlier work of Sugimoto [12], Bettess [13] and Gordon [14. By using the divergence theorem Gabard [15] addresses the issue of integrating general polynomial-exponential products by deriving exact expressions for these integrals in the case where the domain of integration is polygonal. The author applies the technique to the calculation of PUFEM triangular elements. Following a somewhat different approach El Kacimi [16 presents an exact integration scheme for the evaluation of PUFEM element matrices for elastic wave scattering problems in two dimensions. In this work successive integration by parts is performed to obtain closed forms that do not require the coefficients of the polynomial function of the integrand, unlike in reference [15. More recently, a semi-analytical procedure to efficiently integrate the product of a smooth function and a complex exponential over tetrahedral elements has been published in 17. The method allows to consider a wider class of smooth functions, i.e. non-polynomial, but remains limited to exponentials with arguments depending linearly with geometrical coordinates.

The purpose of this paper is to bring new contribution to the PUFEM technique for the simulation of acoustic fields in 3D domains. In particular, a new exact integration scheme over linear tetrahedral elements is presented. Through successive use of Green's theorem, it is shown that volume integrals have closed-form expressions in which no numerical integration is involved. Some numerical aspects of this integration algorithm are also discussed and illustrated. The efficiency of the PUFEM method, in terms of data reduction, is shown and a guideline for selecting the number of plane waves attached to each nodes of the mesh is proposed.

The paper is organized as follows: after presenting the general PUFEM formulation with plane waves in Section 2, the derivation of an exact integration algorithm for the computation of element matrices is explained in Section 3. A convergence anaysis is performed in Section 4. It is shown that PUFEM elements allow to capture accurately the wave field with a number of degrees of freedom that only grows quadratically with the frequency, yielding drastic data reduction compared to classical FEM. The case of a singularity (radiating monopole) in the computational domain is also investigated. The paper finishes with applications of practical interest in Section 5 for the case of a monopole sound source placed in a reverberation room with locally reacting walls.

\section{PUFEM formulation}

For the time harmonic wave oscillation problem, where time-dependence $\mathrm{e}^{-\mathrm{i} \omega t}$ is assumed, the homogeneous Helmholtz equation for the acoustic pressure $p$ in the bounded three-dimensional domain $\Omega$ is

$$
\Delta p+k^{2} p=0 \quad \text { in } \Omega
$$

where $k=\frac{\omega}{c}$ is the wavenumber and $\omega$ is the angular frequency. In this work, we assume that the pressure satisfies the local boundary condition of Robin type:

$$
\frac{\partial p}{\partial n}=\hat{\alpha} p+\hat{\beta}
$$

where $\hat{\alpha}$ and $\hat{\beta}$ are complex-valued functions defined on the boundary $\Gamma=\partial \Omega$. Applying the standard weighted residual scheme to the governing equation, we can write

$$
\int_{\Omega} w\left(\Delta p+k^{2} p\right) \mathrm{d} \Omega=0,
$$


where $w$ stands for the weighting function. The choice of this function is not unique. Among them, the Galerkin method which is probably the most common approach adopted in the finite element community, consists in choosing the weighting function to be identical to the trial basis function. The reason of its popularity among others stems from the fact that this selection allows the construction of a symmetric linear system. By applying integration by parts, the weak form of the Helmholtz equation becomes

$$
\int_{\Omega}\left(\nabla p \cdot \nabla w-k^{2} p w\right) \mathrm{d} \Omega-\int_{\Gamma} \hat{\alpha} p w \mathrm{~d} \Gamma=\int_{\Gamma} \hat{\beta} w \mathrm{~d} \Gamma
$$

The computational domain is now partitioned as $\Omega=\bigcup_{e=1}^{N} \Omega_{e}$, in which $N$ denotes the number of partitioned element of the whole domain. Like standard FEM, each element is parameterized classically via Lagrange interpolation

$$
\boldsymbol{x} \in \Omega_{e} \quad \Leftrightarrow \quad \boldsymbol{x}=\sum_{j=1}^{J} N_{j}\left(\zeta_{1}, \zeta_{2}, \zeta_{3}\right) \boldsymbol{x}_{j},
$$

in which $N_{j}$ is the Lagrangian shape function with regard to the $j^{\text {th }}$ node of the element $\Omega_{e}$, call it $\boldsymbol{x}_{j}$, and $\left(\zeta_{1}, \zeta_{2}, \zeta_{3}\right)$ are the coordinates in the reference frame. Following PUFEM discretization procedure, the sound pressure field is approximated in each element $\Omega_{e}$ as

$$
p(\boldsymbol{x})=\sum_{j=1}^{J} N_{j} P_{j}(\boldsymbol{x}),
$$

In classical FEM, functions $P_{j}$ are simply the value of the pressure at node $\boldsymbol{x}_{j}$, the key idea of the PUFEM relies on the enrichment of the conventional finite element approximation by including solutions of the homogeneous partial differential equation. In the Helmholtz case plane waves, circular waves (Bessel functions) and wavebands are commonly used [5,15-17]. In this work, plane waves are chosen as they permit to develop fast integration algorithms, as shown in the next section. We introduce the set of $Q_{j}$ plane waves as

$$
P_{j}(\boldsymbol{x})=\sum_{q=1}^{Q_{j}} A_{j q} \exp \left(\mathrm{i} k \boldsymbol{d}_{j q} \cdot \boldsymbol{x}\right),
$$

where $\boldsymbol{d}_{j q}$ denotes the wave direction which belongs to the unit sphere, i.e. $\left\|\boldsymbol{d}_{j q}\right\|=1$, and the unknown coefficient $A_{j q}$ stands for the amplitude of the $q$-th plane wave attached to node $j$. Figure 1 illustrates the set of plane waves at node $x_{j}$ of element $\Omega_{e}$ (a tetrahedral element with four node is shown here).

We now proceed to derive the PUFEM matrix $A_{e}$ associated with a single element $\Omega_{e}$. From (6) and (7), both trial and test functions are of the following form:

$$
p=N_{j} \exp \left(\mathrm{i} k \boldsymbol{d}_{j q} \cdot \boldsymbol{x}\right) \quad \text { and } \quad w=N_{j^{\prime}} \exp \left(\mathrm{i} k \boldsymbol{d}_{j^{\prime} q^{\prime}} \cdot \boldsymbol{x}\right) .
$$

Substituting (8) in the weak form of the governing Helmholtz equation (4), we arrive at PUFEM matrix coefficients which are of the form:

$$
\begin{aligned}
\left(\mathrm{A}_{e}\right)_{j q, j^{\prime} q^{\prime}} & =\int_{\Omega_{e}}\left(\nabla p \cdot \nabla w-k^{2} p w\right) \mathrm{d} \Omega \\
& =-k^{2}\left(1+\boldsymbol{d}_{j q} \cdot \boldsymbol{d}_{j^{\prime} q^{\prime}}\right) \int_{\Omega_{e}} N_{j} N_{j^{\prime}} \phi \mathrm{d} \Omega+\mathrm{i} k \boldsymbol{d}_{j^{\prime} q^{\prime}} \cdot \int_{\Omega_{e}} \nabla N_{j} N_{j^{\prime}} \phi \mathrm{d} \Omega \\
& +\mathrm{i} k \boldsymbol{d}_{j q} \cdot \int_{\Omega_{e}} \nabla N_{j^{\prime}} N_{j} \phi \mathrm{d} \Omega+\int_{\Omega_{e}} \nabla N_{j} \cdot \nabla N_{j^{\prime}} \phi \mathrm{d} \Omega,
\end{aligned}
$$

where, function $\phi$ is also a plane wave stemming from the product of the trial and test functions. This can be written as

$$
\phi=\exp (\mathrm{i} \kappa \boldsymbol{d} \cdot \boldsymbol{x}) \quad \text { with } \quad \kappa=k\left\|\boldsymbol{d}_{j q}+\boldsymbol{d}_{j^{\prime} q^{\prime}}\right\| \quad \text { and } \quad \boldsymbol{d}=\frac{\boldsymbol{d}_{j q}+\boldsymbol{d}_{j^{\prime} q^{\prime}}}{\left\|\boldsymbol{d}_{j q}+\boldsymbol{d}_{j^{\prime} q^{\prime}}\right\|}
$$




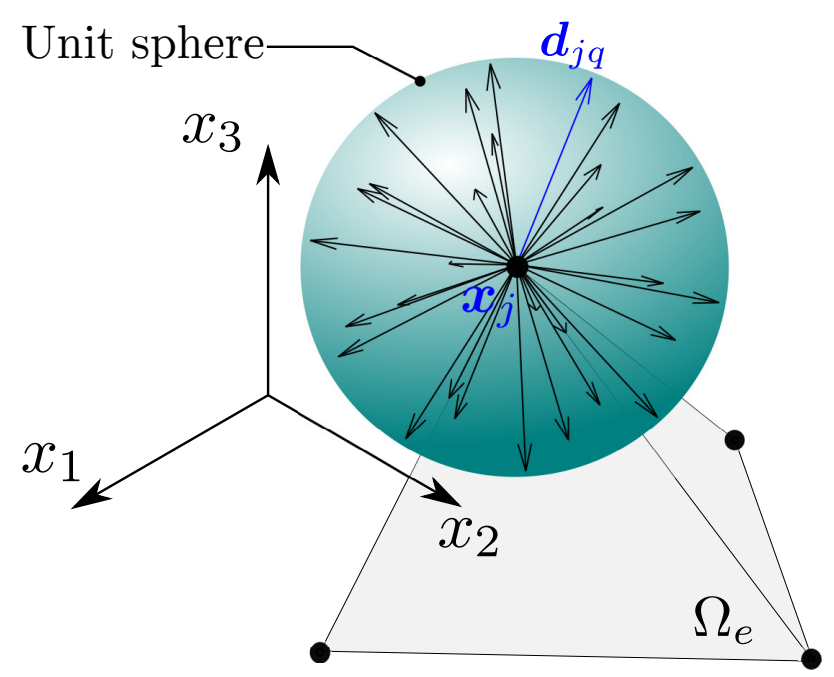

Figure 1: Distribution of 30 plane wave directions (resulting from the Coulomb force algorithm) attached to node $x_{j}$.

Note that for all quantities defined in (10) we omitted the dependence in $j, q, j^{\prime}$ and $q^{\prime}$ for the sake of clarity.

As for the choice of the wave directions, these are chosen to be regularly distributed over the unit circle in the bidimensional case. In three dimensions, in order to ensure that the distribution of plane wave directions is also based on the equal spacing of points on the unit sphere, we apply the Coulomb force method developed by [18. It is based on the use of an explicit time stepping scheme to converge to a static equilibrium state for a set of charged particles on a unit spherical surface. Compared to the discretized cube method used in [8], the use of Coulomb force method allows to obtain uniformly spaced directions, and at the same time, avoid the possibility of a subset of plane waves gathering together in a preferred direction which might deteriorate the stability of the PUFEM solution. The other advantage of the Coulomb force method is that an arbitrary number of wave directions can be assigned, which renders the PUFEM method more flexible. The computational aspect of the Coulomb force method is given in detail in [18 and this will not be repeated here. The special case in (10) when directions of waves are opposite, i.e. $\kappa=0$, is avoided by adding small random variations, such that two wave directions will never be exactly opposite to each other. Finally, once a particular set of directions has been found, it is stored once for all. For the sake of illustration, the distribution of 30 plane wave directions is given in Fig. 1

\section{Exact integration algorithm for linear transformation}

At this point, it is imperative to develop an efficient three-dimensional integration algorithm for addressing the computation of 3D highly oscillatory wave integrals involved in (9). Typically, the computational burden stemming from the huge amounts of integration Gauss points, which is expected to grow cubically with $k$, will be inevitable if standard quadrature methods are used. To make some progress, we consider a linear mapping system between real and local coordinate, as shown in Figure 2. The linear shape functions are of the form: $N_{1}=\zeta_{1}, N_{2}=\zeta_{2}, N_{3}=\zeta_{3}$ and $N_{4}=1-\zeta_{1}-\zeta_{2}-\zeta_{3}$, and we can write explicitly the linear mapping between real space $\boldsymbol{x}=\left(x_{1}, x_{2}, x_{3}\right)$ and local space $\left(\zeta_{1}, \zeta_{2}, \zeta_{3}\right)$ as

$$
\boldsymbol{x}=\sum_{j=1}^{4} N_{j} \boldsymbol{x}_{j}=\boldsymbol{x}_{4}+\zeta_{1} \boldsymbol{e}_{41}+\zeta_{2} \boldsymbol{e}_{42}+\zeta_{3} \boldsymbol{e}_{43} .
$$

Here, we adopt the notation $\boldsymbol{e}_{j j^{\prime}}=\boldsymbol{x}_{j^{\prime}}-\boldsymbol{x}_{j}$, so that the Jacobian matrix can be written by

$$
\mathbf{J}=\left[\begin{array}{lll}
\boldsymbol{e}_{41} & \boldsymbol{e}_{42} & \boldsymbol{e}_{43}
\end{array}\right]^{\top} \text {. }
$$




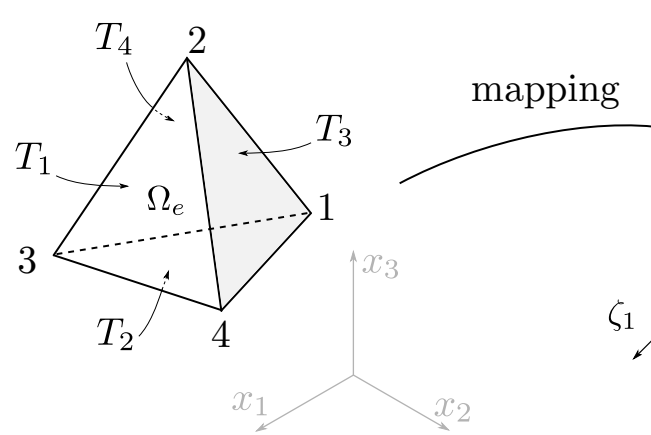

a) real space
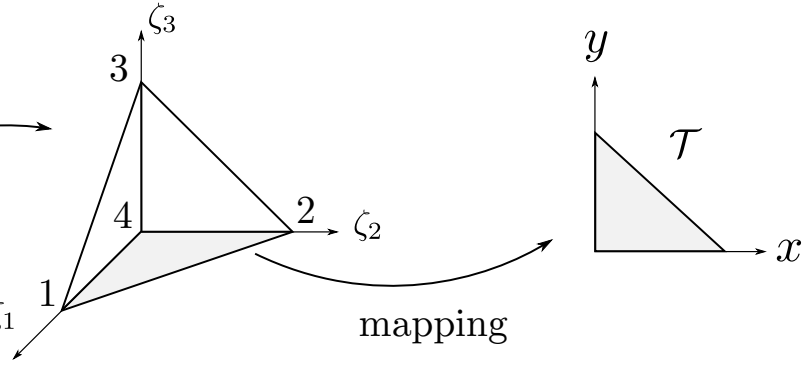

b) local element space

Figure 2: $3 \mathrm{D}$ coordinates systems in real and local element space. The boundary of the $3 \mathrm{D}$ element $\Omega_{e}$ is made of 4 triangular surfaces $T_{\alpha}$ which are all parameterized via the local coordinate system $(x, y)$ defined over the unit triangle $\mathcal{T}$.

Its inverse $\mathbf{H}=\mathbf{J}^{-1}$ can be easily calculated, which allows us to get the transformation formulas for the gradient and Laplace operators:

$$
\nabla F=\mathbf{H} \nabla_{\zeta} F \quad \text { and } \quad \triangle F=H_{i l} H_{i j} \partial_{\zeta_{l} \zeta_{j}} F .
$$

Since the geometric mapping is linear and the shape functions $N_{i}$ are also linear, some terms can be moved out of the integrals in (9), which results in

$$
\begin{aligned}
\left(\mathrm{A}_{e}\right)_{j q, j^{\prime} q^{\prime}} & =-k^{2}\left(1+\boldsymbol{d}_{j q} \cdot \boldsymbol{d}_{j^{\prime} q^{\prime}}\right) \int_{\Omega_{e}} N_{j} N_{j^{\prime}} \phi \mathrm{d} \Omega+\mathrm{i} k \boldsymbol{d}_{j^{\prime} q^{\prime}} \cdot \nabla N_{j} \int_{\Omega_{e}} N_{j^{\prime}} \phi \mathrm{d} \Omega \\
& +\mathrm{i} k \boldsymbol{d}_{j q} \cdot \nabla N_{j^{\prime}} \int_{\Omega_{e}} N_{j} \phi \mathrm{d} \Omega+\nabla N_{j} \cdot \nabla N_{j^{\prime}} \int_{\Omega_{e}} \phi \mathrm{d} \Omega .
\end{aligned}
$$

All integrals involved are of the form

$$
\int_{\Omega_{e}} F \phi \mathrm{d} \Omega
$$

where function $F$ is either quadratic (regardless of the coordinate system, real or local), $F=N_{j} N_{j^{\prime}}$, linear $F=N_{j}$ or simply a constant $F=1$. We can take advantage of the fact that $\phi$ is a plane wave and apply the Green theorem iteratively (see Appendix A). This yields the series of surface integrals

$$
\int_{\Omega_{e}} F \phi \mathrm{d} \Omega=-\int_{S_{e}}\left(\epsilon F \boldsymbol{d}+\epsilon^{2} \nabla F+\epsilon^{3} \triangle F \boldsymbol{d}+\cdots\right) \cdot \boldsymbol{n} \phi \mathrm{d} S,
$$

where $\epsilon=\mathrm{i} / \kappa$ and $S_{e}=\partial \Omega_{e}$. Though the series contains, in principle, an infinite number of terms, the important feature of the linear mapping signifies that the maximum order of the polynomial $F$ in the above volume integrals is quadratic, so only the first three terms of the series are kept. Note that the above equation can be regarded as an alternative formulation to previous research work (see Section 4.1 in [16]). In the following, we define the notation:

$$
S_{e}=\bigcup_{\alpha=1}^{4} T_{\alpha}
$$


to represent all surfaces of the tetrahedron element, where the triangle $T_{\alpha}$ denotes one surface of the element, here $\alpha$ stands for the node number of the element which lies opposite to the surface. Applying (16), we get

$$
\begin{array}{r}
\int_{\Omega_{e}} N_{j} N_{j^{\prime}} \phi \mathrm{d} \Omega=-\sum_{\alpha=1}^{4} \boldsymbol{d} \cdot \boldsymbol{n}_{\alpha}\left(\epsilon \int_{T_{\alpha}} N_{j} N_{j^{\prime}} \phi \mathrm{d} S+\epsilon^{3} \triangle\left(N_{j} N_{j^{\prime}}\right) \int_{T_{\alpha}} \phi \mathrm{d} S\right) \\
-\epsilon^{2} \sum_{\alpha=1}^{4} \boldsymbol{n}_{\alpha} \cdot \nabla N_{j^{\prime}} \int_{T_{\alpha}} N_{j} \phi \mathrm{d} S-\epsilon^{2} \sum_{\alpha=1}^{4} \boldsymbol{n}_{\alpha} \cdot \nabla N_{j} \int_{T_{\alpha}} N_{j^{\prime}} \phi \mathrm{d} S,
\end{array}
$$

and

$$
\int_{\Omega_{e}} N_{j} \phi \mathrm{d} \Omega=-\sum_{\alpha=1}^{4} \boldsymbol{d} \cdot \boldsymbol{n}_{\alpha} \epsilon \int_{T_{\alpha}} N_{j} \phi \mathrm{d} S-\epsilon^{2} \sum_{\alpha=1}^{4} \boldsymbol{n}_{\alpha} \cdot \nabla N_{j} \int_{T_{\alpha}} \phi \mathrm{d} S
$$

and

$$
\int_{\Omega_{e}} \phi \mathrm{d} \Omega=-\sum_{\alpha=1}^{4} \boldsymbol{d} \cdot \boldsymbol{n}_{\alpha} \epsilon \int_{T_{\alpha}} \phi \mathrm{d} S .
$$

Here, $n_{a} l p h a$ are outward-pointing normal vectors. At this stage, we shall operate an appropriate change of variable from the real space $T_{\alpha}$ to the local coordinate system $(x, y)$ defined over the unit triangle $\mathcal{T} \equiv\{x \geq 0, y \geq 0, x+y \leq 1\}$. By definition, the node $\boldsymbol{x}_{\alpha}$ does not belong to $T_{\alpha}$. Thus, the set $T_{\alpha}$ can be defined using the three other nodes and we can put

$$
\boldsymbol{x} \in T_{\alpha} \quad \Leftrightarrow \quad \boldsymbol{x}=\boldsymbol{x}_{\alpha-1}+x\left(\boldsymbol{x}_{\alpha+1}-\boldsymbol{x}_{\alpha-1}\right)+y\left(\boldsymbol{x}_{\alpha+2}-\boldsymbol{x}_{\alpha-1}\right)
$$

with the convention that $\boldsymbol{x}_{\alpha+4}=\boldsymbol{x}_{\alpha}$. So, if we define $\omega_{\alpha}=\mathrm{i} \kappa \boldsymbol{d} \cdot \boldsymbol{x}_{\alpha}$, we can calculate

$$
\begin{aligned}
\int_{T_{\alpha}} \phi \mathrm{d} S & =\int_{T_{\alpha}} \exp \left[\mathrm{i} \kappa \boldsymbol{d} \cdot\left(\boldsymbol{x}_{\alpha-1}+x\left(\boldsymbol{x}_{\alpha+1}-\boldsymbol{x}_{\alpha-1}\right)+y\left(\boldsymbol{x}_{\alpha+2}-\boldsymbol{x}_{\alpha-1}\right)\right)\right] \mathrm{d} S \\
& =2 A_{\alpha} \mathrm{e}^{\omega_{\alpha-1}} \int_{\mathcal{T}} \exp \left[\left(\omega_{\alpha+1}-\omega_{\alpha-1}\right) x+\left(\omega_{\alpha+2}-\omega_{\alpha-1}\right) y\right] \mathrm{d} x \mathrm{~d} y,
\end{aligned}
$$

where $A_{\alpha}$ is the aera of $T_{\alpha}$. Now, it is useful for the analysis to introduce the family of surface integrals

$$
I_{m n}(\boldsymbol{a})=\int_{\mathcal{T}} x^{m} y^{n} \hat{\phi} \mathrm{d} x \mathrm{~d} y,
$$

where $\hat{\phi}=\exp (a x+b y)$ and $\boldsymbol{a}=(a, b)$ is an arbitrary vector. Thus, we can write

$$
\int_{T_{\alpha}} \phi \mathrm{d} S=2 A_{\alpha} \mathrm{e}^{\omega_{\alpha-1}} I_{00}(\boldsymbol{a}) \quad \text { with } \quad \boldsymbol{a}=\left(\omega_{\alpha+1}-\omega_{\alpha-1}, \omega_{\alpha+2}-\omega_{\alpha-1}\right) .
$$

It is also convenient to introduce a set of integers $\mathcal{S}$ which contains the four node numbers associated with the element, that is $\mathcal{S}=\{1,2,3,4\}$. Following the same approach, it can be shown that

$$
\int_{T_{\alpha}} N_{j} \phi \mathrm{d} S=2 A_{\alpha} \mathrm{e}^{\omega_{r}} I_{10}(\boldsymbol{a}) \text { with } \boldsymbol{a}=\left(\omega_{j}-\omega_{r}, \omega_{l}-\omega_{r}\right),
$$

where it must be understood that the set of indices $\alpha, j, l$ and $r$ must constitute the set $\mathcal{S}$, namely, $\{\alpha, j, l, r\}=\mathcal{S}$, which signifies that $\alpha, j, l, r$ must be all different. Otherwise, the value of the integral is zero (this happens when $\alpha=j$ ). Similarly

$$
\int_{T_{\alpha}} N_{j} N_{j^{\prime}} \phi \mathrm{d} S=2 A_{\alpha} \mathrm{e}^{\omega_{r}} I_{11}(\boldsymbol{a}) \quad \text { with } \quad \boldsymbol{a}=\left(\omega_{j}-\omega_{r}, \omega_{j^{\prime}}-\omega_{r}\right),
$$


where it must be understood that $\left\{j, j^{\prime}, \alpha, r\right\}=\mathcal{S}$. Otherwise, the value of the integral is zero (this happens when $\alpha=j$ or $\alpha=j^{\prime}$ ). For the particular case where $j=j^{\prime}$, we have

$$
\int_{T_{\alpha}} N_{j}^{2} \phi \mathrm{d} S=2 A_{\alpha} \mathrm{e}^{\omega_{r}} I_{20}(\boldsymbol{a}) \quad \text { with } \quad \boldsymbol{a}=\left(\omega_{j}-\omega_{r}, \omega_{l}-\omega_{r}\right) .
$$

The numerical treatment of 23 is presented with all necessary details in Appendix B.

We shall mention exceptional cases that might lead to wrong results corrupted by round-off errors. The first one corresponding to $\kappa \approx 0$ is easily circumvented by selecting plane wave directions accordingly (see previous section). A second scenario occurs when $\boldsymbol{a} \approx 0$ in equation $(45)$, this case characterizes the fact that the resulting plane wave direction is orthogonal to one of the surfaces of the element. To tackle this problem, we shall take advantage of the fact that the exponential function $\hat{\phi}$ is nearly a constant so quadrature methods are well suited. Integrals are computed numerically using the discrete sum

$$
\int_{\mathcal{T}} f(x, y) \mathrm{d} x \mathrm{~d} y \approx \sum_{g} w_{g} f\left(x_{g}, y_{g}\right),
$$

where $w_{g}$ and $\left(x_{g}, y_{g}\right)$ are, respectively the weight and the quadrature point on the unit triangle. In practice, the summation is performed over a very small number of terms to achieve high accuracy and the condition $\boldsymbol{a} \approx 0$ means $\|\boldsymbol{a}\|<10^{-2}$. The use of closed-form expressions also break down when $a \approx 0$ or $b \approx 0$ or $s \approx 0$ in Eq. (48), this case signifies that the resulting plane wave direction is orthogonal to one edge of the surface integration domain. Again, the use of Gauss quadrature allows to obtain extremely accurate and reliable results.

\section{Note on computational efficiency}

The complexity of the integration algorithm can be assessed for the case of single element having $Q_{j}$ plane wave directions 'attached' to each node $\boldsymbol{x}_{j}$. Thus, $Q_{j} \times Q_{j^{\prime}}$ corresponds to the total number of plane waves (i.e. function $\phi$ ) involved for a given pair of indices $\left(j, j^{\prime}\right)$. The computational cost depends largely on the number of (non-zero) surface integrals and the number of blocks $\mathrm{B}_{\left(j, j^{\prime}\right)}$ (of size $Q_{j} \times Q_{j^{\prime}}$ ) in the PUFEM elementary matrix which is of the form:

$$
\mathrm{A}_{e}=\left(\begin{array}{cccc}
\mathrm{B}_{(1,1)} & \mathrm{B}_{(1,2)} & \mathrm{B}_{(1,3)} & \mathrm{B}_{(1,4)} \\
" & \mathrm{~B}_{(2,2)} & \mathrm{B}_{(2,3)} & \mathrm{B}_{(2,4)} \\
" & " & \mathrm{~B}_{(3,3)} & \mathrm{B}_{(3,4)} \\
" & " & & \mathrm{~B}_{(4,4)}
\end{array}\right) .
$$

and for which only 6 off-diagonal and 4 diagonal blocks need computing as the rest is obtained by invoking symmetry. The implemented version of the algorithm follows the description presented earlier : the volume integral is converted into surface integrals over the reference triangle and then converted into line integrals for which closed-form solutions are available. To speed up the integration, a Fortran version has been implemented and linked as a mexfile to Matlab core. This version takes advantage of multithread capability of modern CPU with openMP shared memory parallelism. The double loop over the tetrahedron vertices is distributed over the available threads.

As illustrated in Fig. 3, for a sufficiently large number of plane waves (say $Q>150$, here we took $Q_{j}=Q$ ), the scaling is almost linear up to 5 threads. The use of a larger number of threads appears to be inefficient due to the overhead. A profile (valgrind/cachegrind) indicates that around $15 \%$ of CPU time is spent for the computation. The reminder of the computational time is spend mostly for the recombination.

\section{Convergence analysis}

In this section, we shall investigate the numerical performance of the PUFEM in terms of accuracy and data reduction. Here the idea is to test the convergence of the method without modifying the coarse mesh. 


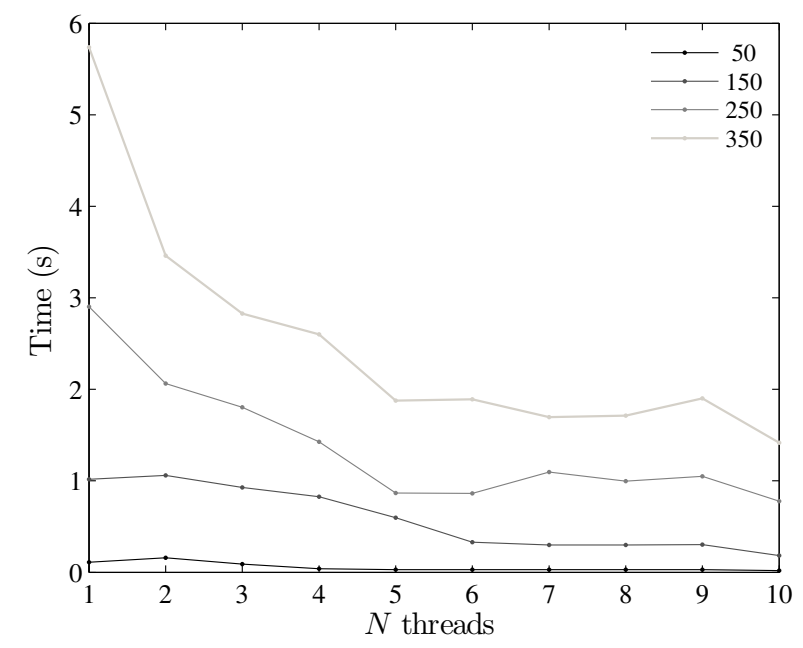

Figure 3: Running time for computing a single elementary PUFEM matrix with different number of plane waves $Q=50,150,250$ and 350. Test ran on $4 \operatorname{Intel}(\mathrm{R})$ Xeon(R) CPU E5-2670 @ 2.60GHz with 16 cores and 32 threads available.

In other words, we perform a $Q$-refinement as opposed to a $h$-refinement. Following theoretical arguments given in 20] and 21] on acoustic field synthesis and the Fast Multipole method, we may assume that the number of plane waves attached to each node of the FEM mesh should vary quadratically as

$$
Q_{\text {node }}=C(k \bar{h})^{2} .
$$

This criteria which is also consistent with results given in [6] is node-dependent and $\bar{h}$ is the longest edge attached to the node of the mesh. In practice, coefficient $C$ must also be viewed as a function of $k \bar{h}$ and it must be adjusted depending on the configuration and the expected accuracy. The behavior of $C$ with respect to the non-dimensional frequency $k \bar{h}$ can be found by taking advantage of an artificial wave propagation problem for which the analytical solution is easily available. To do this, we consider an arbitrary incident plane wave $p^{i n c}$ propagating inside a single regular tetrahedron (the 4 edges are all of equal length $h=\bar{h}$ ). The boundary condition applied on each face is given by

$$
\frac{\partial p}{\partial n}=\mathrm{i} k\left(p-p^{i n c}\right)+\frac{\partial p^{i n c}}{\partial n} .
$$

To be fair in our convergence test, the incident plane wave direction is always chosen as far as possible from the plane waves directions of the PUFEM basis, so that peculiar behaviors can be avoided. In all cases, plane wave directions are computed through the algorithm presented in Section 2, with the aim to ensure a regularly spaced distribution. The numerical error is evaluated via $L_{2}$ error criteria on the boundary of the domain (here we have simply: $\Gamma=\partial \Omega_{e}$, as there is a single element, and $p^{e x}=p^{i n c}$ ):

$$
\varepsilon_{2}=\frac{\left\|p^{e x}-p\right\|_{L_{2}(\Gamma)}}{\left\|p^{e x}\right\|_{L_{2}(\Gamma)}}
$$

In Table 11. convergence results are reported. All these tests have been carried out to assess the convergence and verify the effectiveness of the method. Here the condition number Cond is estimated with Matlab by using the command Condest. Moreover, we also introduce the average discretization level $n_{\lambda}$ in the context of $3 \mathrm{D}$ PUFEM, $n_{\lambda}$ describes the number of variables needed to capture a single wavelength. For $3 \mathrm{D}$ problems, it is evaluated via:

$$
n_{\lambda}=\lambda\left(\frac{N_{d o f}}{\int_{\Omega_{e}} \mathrm{~d} \Omega}\right)^{1 / 3} .
$$




\begin{tabular}{ccccccc}
\hline$k h$ & $C$ & $Q$ & $n_{\lambda}$ & $h / \lambda$ & $\log _{10}($ Cond $)$ & $\varepsilon_{2}(\%)$ \\
\hline 20 & 0.080 & 32 & 2.04 & 3.18 & 6.99 & 86.59 \\
& 0.130 & 52 & 2.39 & 3.18 & 9.24 & 9.21 \\
& 0.180 & 72 & 2.67 & 3.18 & 11.53 & 0.42 \\
& 0.230 & 92 & 2.89 & 3.18 & 14.26 & 0.020 \\
& 0.280 & 112 & 3.09 & 3.18 & 16.44 & 0.0036 \\
& 0.300 & 120 & 3.16 & 3.18 & 17.70 & 0.0057 \\
\hline 45 & 0.090 & 183 & 1.62 & 7.16 & 10.72 & 2.82 \\
& 0.105 & 213 & 1.70 & 7.16 & 12.37 & 0.64 \\
& 0.120 & 243 & 1.78 & 7.16 & 14.07 & 0.077 \\
& 0.135 & 273 & 1.85 & 7.16 & 16.19 & 0.018 \\
& 0.150 & 303 & 1.91 & 7.16 & 17.67 & 0.0027 \\
& 0.164 & 333 & 1.98 & 7.16 & 19.23 & 0.0025 \\
\hline 65 & 0.085 & 360 & 1.40 & 10.35 & 13.12 & 9.41 \\
& 0.094 & 395 & 1.45 & 10.35 & 14.33 & 0.19 \\
& 0.102 & 430 & 1.49 & 10.35 & 16.39 & 0.037 \\
& 0.110 & 465 & 1.53 & 10.35 & 17.22 & 0.018 \\
& 0.118 & 500 & 1.56 & 10.35 & 18.68 & 0.0027 \\
& 0.127 & 535 & 1.60 & 10.35 & 19.20 & 0.0029 \\
\hline 80 & 0.080 & 513 & 1.28 & 12.73 & 14.09 & 1.08 \\
& 0.086 & 549 & 1.31 & 12.73 & 15.30 & 0.29 \\
& 0.091 & 585 & 1.34 & 12.73 & 16.28 & 0.092 \\
& 0.097 & 621 & 1.37 & 12.73 & 17.29 & 0.011 \\
& 0.103 & 657 & 1.39 & 12.73 & 18.75 & 0.0021 \\
& 0.108 & 693 & 1.42 & 12.73 & 19.78 & 0.0018 \\
\hline
\end{tabular}

Table 1: Convergence test of the PUFEM for a single element of size $h$.

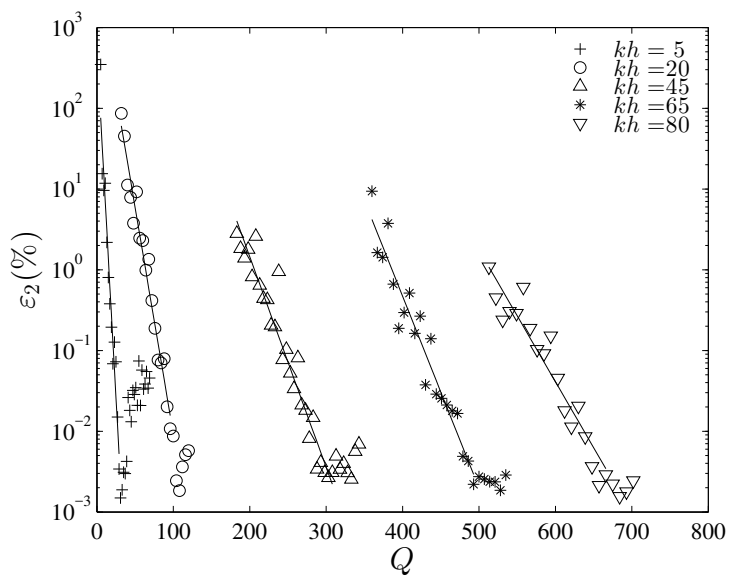

(a)

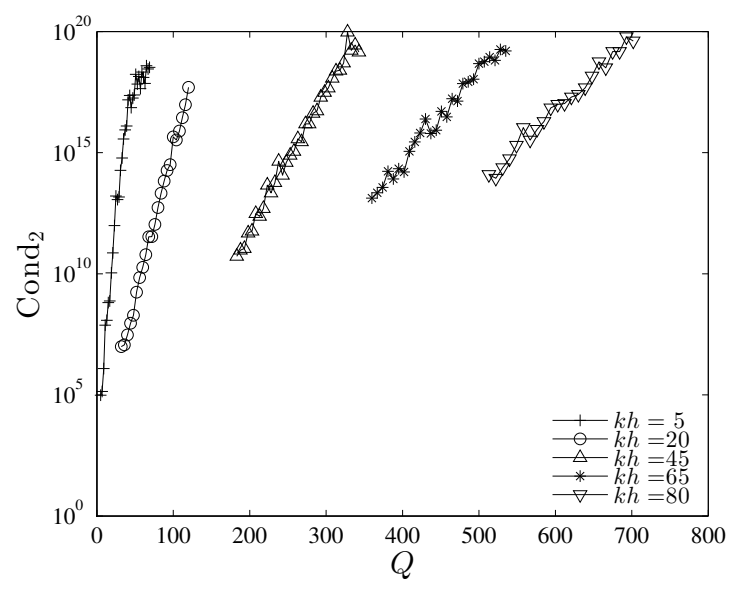

(b)

Figure 4: The relative error (a), condition number (b), with respect to $k h$. 


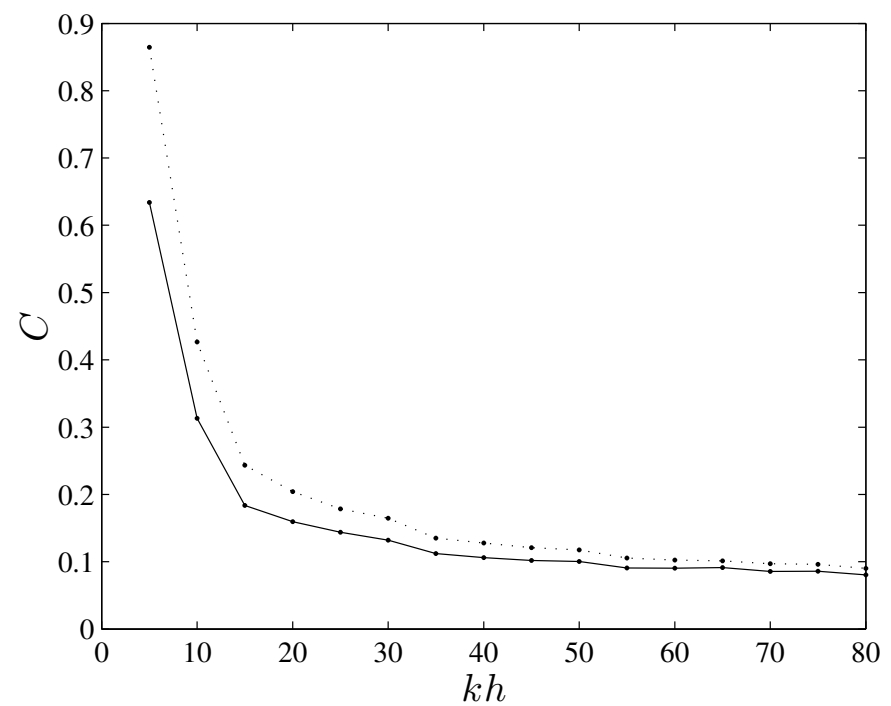

Figure 5: Coefficient $C$ with respect to $k h$ for an expected accuracy of $1 \%$ (dotted) and $0.1 \%$ (straight).

where $N_{d o f}$ is the total number of degrees of freedom.

After running a series of numerical tests, we managed to obtain the error performance with respect to different values of the non-dimensional parameter $k h$, varying from 5 to 80 . For the sake of clarity, only 5 of them are displayed in Figure 4. We can observe that the region of convergence, once it begins, is relatively narrow, and the error drops abruptly showing exponential rates of convergence until it reaches a plateau. This is mainly caused by the large condition number inherent to PUFEM with plane waves. A closer analysis reveals that the plateau is reached as soon as the condition number exceeds $10^{16}$ or $10^{17}$.

Interestingly, if we draw a horizontal line corresponding to a specified error level, say $1 \%$ for instance, then the intersection points with each curve corresponding to a given value of $k h$ will give the required number of plane wave directions to attain this accuracy. Hence, by inverting formula (30), we can deduce the value of coefficient $C$ that guarantees a certain prescribed accuracy. Values of coefficient $C$ are reported in Figure 5 for two prescribed accuracies of $\varepsilon_{2}=1 \%$ and $\varepsilon_{2}=0.1 \%$. It can be observed that the value of $C$ decreases and converges to a certain value around 0.1 for high frequency. More precisely, the curves show similar asymptotic behavior as $Q \sim 0.1(k h)^{2}$ and the coefficient $C$ ranges between 0.1 and 0.7 as long as the frequency is sufficiently high compared to the element length (i.e., $k h>10$ ). This suggests that an unreasonable number of plane waves are needed for small value of $k h$. In practice, it shows that PUFEM is not efficient enough for the low-frequency scenario when $k h<10$. In this case, the use of classical piecewise polynomial functions would be a better option. For sufficiently large frequency, or more precisely when $k h>10$, the PUFEM clearly outperforms standard piecewise polynomial FEM. To summarize, we can anticipate that, the number of plane waves should vary quadratically with respect to $k h$ whereas the number of degrees of freedom required by standard FEM should grow cubically with frequency, thus the computational gain is expected to be very substantial at high frequency.

Before we end this section, one should have in mind that these results can be regarded as nearly-optimal as the problem treated here consists in approximating an arbitrary propagating plane wave with other plane waves propagating in multiple directions. In the $2 \mathrm{D}$ case, a rigorous analysis can be conducted as in [19, the 3D case appears to be more tricky since the wave directions are not known a priori as these are computed using the Coulomb force method. Finally, the optimal curves of figure 5 should provide a useful 'rule-ofthumb' for assigning the appropriate number of plane waves at each node of the mesh. An example of this shall be given in the next section. 


\section{Applications}

\subsection{Response to a point source in a hard wall cubic cavity}

We now consider the case of radiating monopole placed in a hard wall cubic cavity of size $2 \times 2 \times 2$ (dimensions are given in meters). The total pressure field satisfies

$$
\Delta p+k^{2} p=\delta\left(\boldsymbol{x}-\boldsymbol{x}_{0}\right),
$$

where $\boldsymbol{x}_{0}$ is the source location. The exact solution to this problem can be recovered by the modal series 22 .

$$
p^{e x}=\sum_{m} \frac{\psi_{m}(\boldsymbol{x}) \psi_{m}\left(\boldsymbol{x}_{0}\right)}{k^{2}-k_{m}^{2}}
$$

where $\left(\psi_{m}, k_{m}\right)$ are the normalized cavity modes and the associated frequency. In order to circumvent the singular behavior of the solution, it is judicious to split the pressure as $p=p^{I}+p^{S}$ where

$$
p^{I}=\exp \left(\mathrm{i} k\left|\boldsymbol{x}-\boldsymbol{x}_{0}\right|\right) /\left(4 \pi\left|\boldsymbol{x}-\boldsymbol{x}_{0}\right|\right)
$$

is the free space incident field and $p^{S}$ is the scattered field which is solved with the PUFEM with Neumann boundary conditions: $\partial_{n} p^{S}=-\partial_{n} p^{I}$. The cavity is meshed with 24 elements of typical size $h=2$ with a total of 15 nodes. In Fig. 6, are plotted the PUFEM solution due a point source located near the center with $\boldsymbol{x}_{0}=(0.9,0.8,1)$. Three numerical tests have been ran by increasing the number of plane waves. Note that the selection rule (30) indicates that the number of plane waves differs depending on the type of node it is attached to, thus $Q_{\max }$ refers to the number of plane waves attached to nodes belonging to largest elements, i.e. the eight corners of the cavity. Clearly, PUFEM solutions with $Q \max =278$ have converged to reasonable accuracy. A careful examination of the imaginary part of the computed solution, which is a consequence of the complex-valued plane wave basis, shows that it tends to vanish as the number of plane waves increases. Since the true solution is purely real, the imaginary part could serve as an indicator of the numerical accuracy. In this scenario, we shall take advantage of this and define the following error estimate

$$
\tilde{\varepsilon}_{2}=\frac{\|\Im(p)\|_{L_{2}(\Gamma)}}{\|\Re(p)\|_{L_{2}(\Gamma)}} .
$$

Results of Fig. 7 7 confirm that the two error indicators 32 and (37) are in perfect agreement (note that $\langle Q\rangle$ refers to the average number of plane waves per node used in the calculation). The observed discrepancies when $\langle Q\rangle$ exceeds 300 stem simply from the fact that the truncated modal series does not contain enough terms to guarantee very precise results. Thus the indicator $(37)$ is very reliable and results show similar convergence properties as for the test problem of Figure 4. The third curve shows errors obtained when the total pressure $p$ is computed using a direct approach, that is by expanding the right-hand side of the wave equation in the PUFEM plane wave basis. In this context, the weak form (4) is

$$
\int_{\Omega}\left(\nabla p \cdot \nabla w-k^{2} p w\right) \mathrm{d} \Omega=-\int_{\Omega} w \delta\left(\boldsymbol{x}-\boldsymbol{x}_{0}\right) \mathrm{d} \Omega=-w\left(\boldsymbol{x}_{0}\right) .
$$

This indicates, as expected, that the plane wave basis cannot capture correctly singular or quasi-singular fields.

\subsection{Response to a point source in a treated room}

In this section, the simulation of acoustic fields in a reverberation room with treated walls is considered. The shape of the room is taken from a reverberation chamber from our laboratory in SUPMECA and the upper surface ('ROOF' as indicated in Fig. 8) is treated acoustically. It is assumed that the surface is locally reacting and

$$
\partial_{n} p=\mathrm{i} k Z^{-1} p,
$$



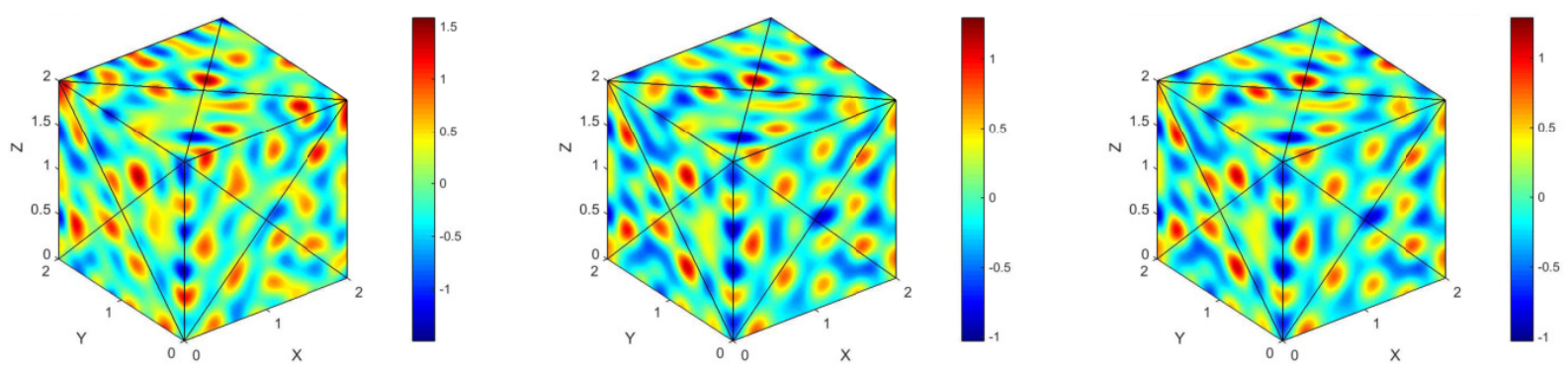

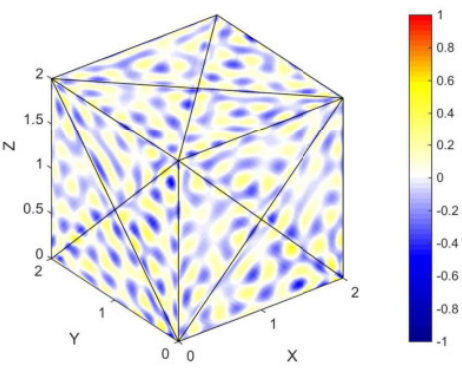

(a) $Q_{\max }=139$

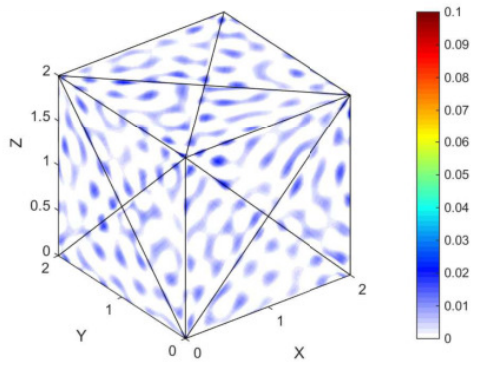

(b) $Q_{\max }=278$

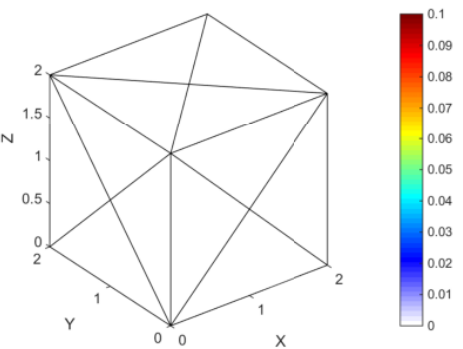

(c) $Q_{\max }=417$

Figure 6: Response in the cavity due to a point source with $k h=30$ : PUFEM solution with $Q \max =139$ (a), $Q_{\max }=278$ (b) and $Q \max =417$ (c). Top: Real part, Bottom: Imaginary part

where $Z$ is the reduced wall impedance. The acoustic monopole source is located at $\boldsymbol{x}_{0}=(0,0,0.5)$.

First, one shall consider a somewhat artificial problem by choosing a purely imaginary wall impedance $Z=2$ i. Figure 9 (left) shows the amplitude of the total pressure field at the specific frequency $f=1620 \mathrm{~Hz}$. Using dimensionless quantities, this corresponds approximately to $k H=60$ where $H \approx 2 \mathrm{~m}$ stands for the longest edge of the mesh, i.e. the height of the room. The number of plane waves at each node of the mesh is chosen using $Q_{\text {node }}=2 C(k \bar{h})^{2}$ (which is twice the 'optimal' case of $(30)$ ) where coefficient $C=C(k \bar{h})$ is node-dependent and chosen from results of Figure 5 . The total number of degrees of freedom is about 12400, which means that the average discretization level is $n_{\lambda}=2.4$ d.o.f. per wavelength. In this scenario, the exact solution is real-valued so it is fair to assume that error estimate (37) is good indicator and it is found that $\tilde{\varepsilon}_{2} \approx 1 \%$ which is sufficiently accurate for engineering purposes. We now repeat the same calculation with a more conventional value for the wall impedance: $Z=2+2$ i. The corresponding acoustic field is illustrated in Figure 9 (right) showing that the wall treatment allows the amplitude of the sound pressure to be reduced by an order of magnitude.

In this last example, we shall consider a cavity problem in the presence of a re-entrant corner, where the sound field is produced by a monopole source placed inside the cavity. In Figure 10 , it can be seen that a small cube of size $1 \times 1 \times 1$ is cut out from the original cubic cavity of dimension $2 \times 2 \times 2$. Similarly to the reverberant room case, a surface impedance boundary condition $Z=2+2 \mathrm{i}$ is also applied on the roof of the cavity, i.e. at $z=2$. The characteristic length of this cavity is $H=2 \mathrm{~m}$. The position of the point source $\boldsymbol{x}_{0}=(0.99,0.99,1.01)$ is located in close proximity of the re-entrant corner of the cavity at a distance of about $d \approx 1.73 \times 10^{-2} \mathrm{~m}$ from the corner. Results (viewed from a given observing angle) are shown in Figure 10 for two discretization levels. In this example, taking an average number of $\langle Q\rangle=220$ plane wave directions per node suffices to guarantee converged results as differences between the two results are hardly noticeable. 


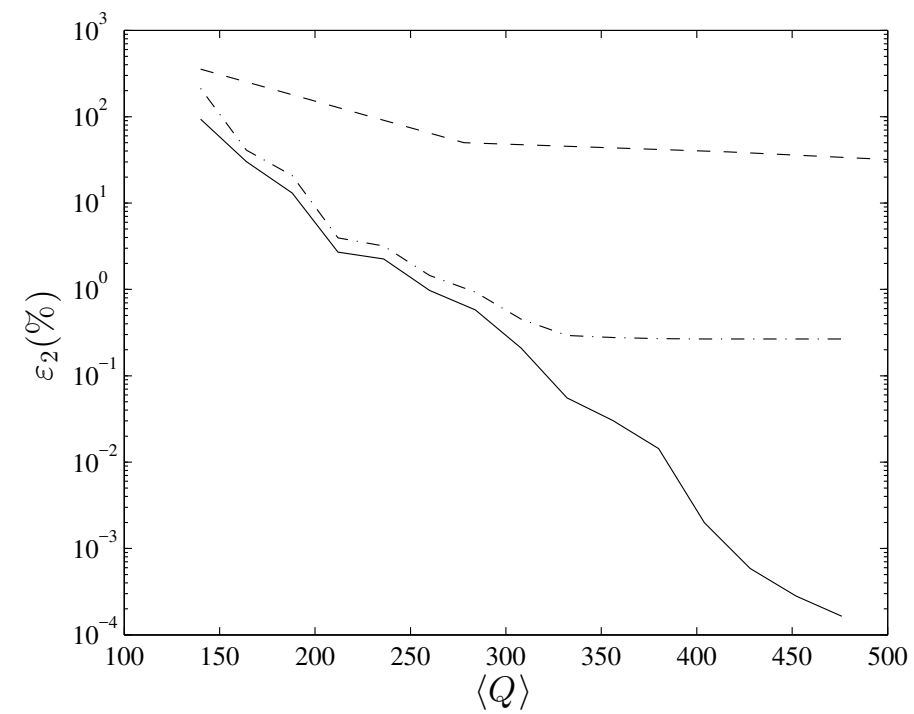

Figure 7: Response in the cavity due to a point source with $k h=30$. Classical error estimate (32) (dotted) and error indicator based on the imaginary part [37) (straight). Dashed line corresponds to results computed using a direct approach.

\section{Conclusions}

This paper has brought new contribution to the PUFEM technique for the simulation of acoustic fields in $3 \mathrm{D}$ domains. In particular, an exact integration scheme is presented for the fast and accurate computation of highly oscillatory integrals arising from the PUFEM matrix coefficients associated with the 3D Helmholtz equation. It is shown that, through successive use of Green's theorem, volume integrals have closed-form expressions in which no numerical integration is involved. Through convergence tests, a criteria for selecting the number of plane waves is proposed. It is shown that this number only grows quadratically with the frequency thus leading to a drastic reduction in the total number of degrees of freedoms in comparison to classical FEM. The method has been verified for two numerical examples. In both cases, the method is shown to converge to the exact solution. For the hard wall cavity problem with a monopole source located inside, a direct approach which consists in projecting the delta function in the plane wave basis shows poor convergence due to the singular nature of the solution. Results of practical interest are shown for the case of a sound source placed in a reverberation room with locally reacting walls. When the surface impedance is purely imaginary the exact solution is real and an error estimate based on the imaginary part of the numerical solution allows to assess the quality of the results at a relatively cheap cost. It is believed that the error estimate could also serve as reliable indicator for arbitrary impedance values. Because the work presented in this paper is still restricted to linear 3D elements, there is a need for more sophisticated integration algorithms able to handle geometrically curved elements and this should be the subject of future work.

\section{Acknowledgements}

This project has been partially funded by the China Scholarship Council. The authors would like to thank Reda Sellakh for providing us with the geometry of the reverberant chamber at SUPMECA. 


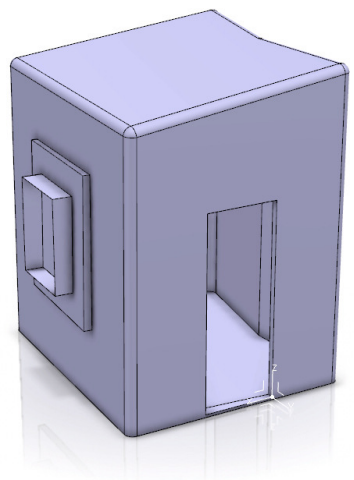

(a)

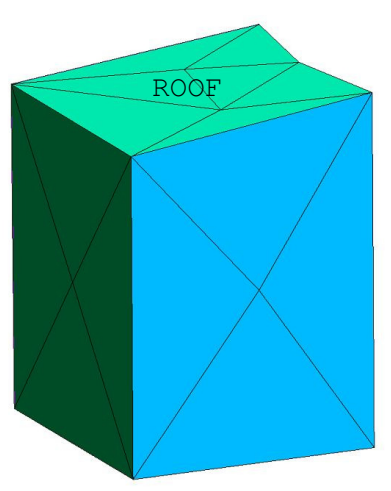

(b)

Figure 8: Geometry of the reverberant chamber at SUPMECA (a) meshed with PUFEM elements (b).

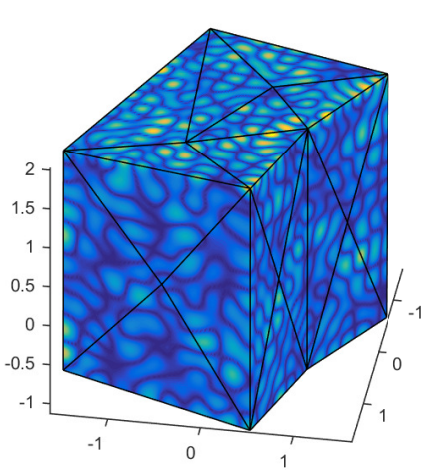

(a)
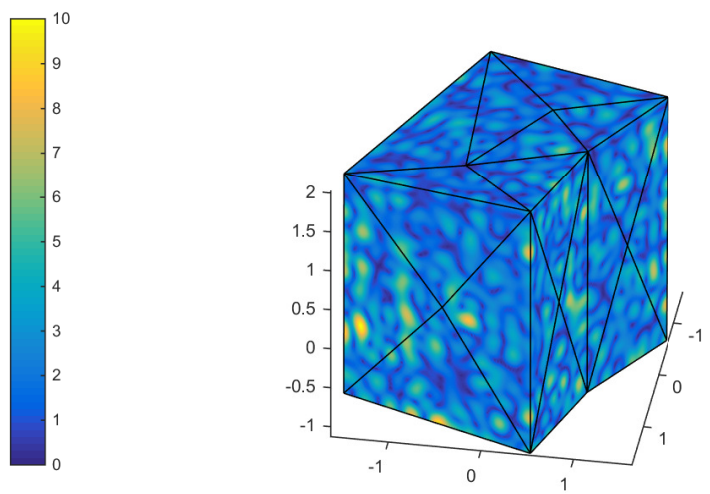

(b)

Figure 9: Amplitude of the total pressure field $|p|$, at $f=1620 \mathrm{~Hz}$. (a): treated roof with purely imaginary impedance $Z=2 \mathrm{i}$ and (b): treated roof with $Z=2+2 \mathrm{i}$.

\section{Appendix A: Green's identity}

Recall that the plane wave $\phi$ satisfies

$$
\nabla \phi=\mathrm{i} \kappa \boldsymbol{d} \phi \quad \text { and } \quad \triangle \phi=-\kappa^{2} \phi
$$

By using second Green's identity,

$$
\int_{\Omega_{e}} F \phi \mathrm{d} \Omega=-\frac{1}{\kappa^{2}} \int_{\Omega_{e}} \triangle F \phi \mathrm{d} \Omega-\frac{1}{\kappa^{2}} \int_{S_{e}}\left(F \partial_{n} \phi-\phi \partial_{n} F\right) \mathrm{d} S .
$$

A similar formula holds by replacing $F$ by $\triangle F$ and it finally yields

$$
\int_{\Omega_{e}} F \phi \mathrm{d} \Omega=-\frac{1}{\kappa^{2}} \int_{S_{e}}\left(F-\frac{1}{\kappa^{2}} \triangle F\right) \partial_{n} \phi-\left(\partial_{n} F-\frac{1}{\kappa^{2}} \partial_{n} \triangle F\right) \phi+\ldots
$$


a)

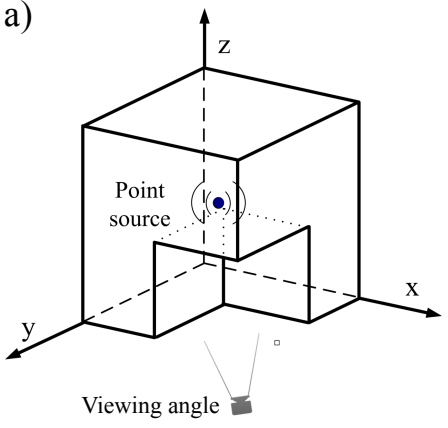

b)

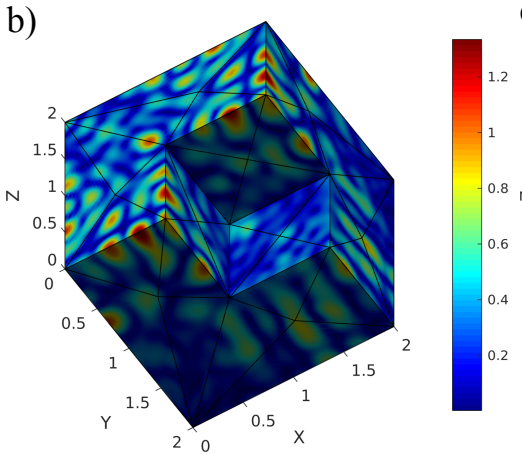

c)

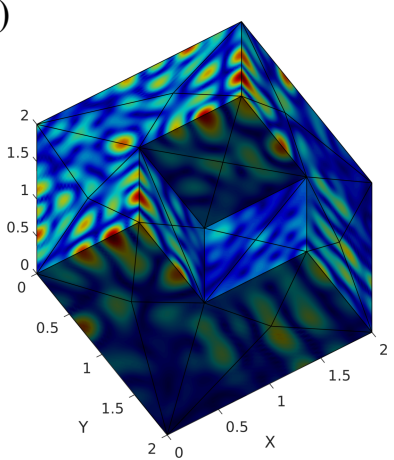

Figure 10: Response to a point source located near a re-entrant corner. (b): $\langle Q\rangle=220$ and (c): $\langle Q\rangle=275$.

Or in a more tractable form (recall that $\epsilon=\mathrm{i} / \kappa)$ :

$$
\int_{\Omega_{e}} F \phi \mathrm{d} \Omega=-\int_{S_{e}}\left(\epsilon F \boldsymbol{d}+\epsilon^{2} \nabla F+\epsilon^{3} \triangle F \boldsymbol{d}+\epsilon^{4} \nabla(\triangle F)+\ldots\right) \cdot \boldsymbol{n} \phi \mathrm{d} S .
$$

\section{Appendix B: Integration over the reference triangle}

The aim is to arrive to a closed-form expression for the exact computation of

$$
I_{m n}(\boldsymbol{a})=\int_{\mathcal{T}} x^{m} y^{n} \hat{\phi} \mathrm{d} x \mathrm{~d} y,
$$

where $\hat{\phi}=\exp (a x+b y)$ and the vector $\boldsymbol{a}=(a, b)$ is complex-valued. In the present context, indices $m, n$ are only varying from 0 to 2 . Figure 11 shows the reference triangular domain. The dimension of the integral domain can be further reduced from surface to its edges by applying Green theorem as in formula (43). This yields

$$
I_{m n}(\boldsymbol{a})=\frac{1}{\|\boldsymbol{a}\|^{2}}[\underbrace{\boldsymbol{a} \cdot \int_{\gamma} x^{m} y^{n} \hat{\phi} \hat{\boldsymbol{n}} \mathrm{d} \gamma}_{I_{m n}^{1}(\boldsymbol{a})}-\underbrace{\int_{\gamma} \nabla\left(x^{m} y^{n}\right) \cdot \hat{\boldsymbol{n}} \hat{\phi} \mathrm{d} \gamma}_{I_{m n}^{2}(\boldsymbol{a})}+\underbrace{\frac{\boldsymbol{a}}{\|\boldsymbol{a}\|^{2}} \cdot \triangle\left(x^{m} y^{n}\right) \int_{\gamma} \hat{\phi} \hat{\boldsymbol{n}} \mathrm{d} \gamma}_{I_{m n}^{3}(\boldsymbol{a})}],
$$

in which, the first line integral can be written in its closed-form expression:

$$
\begin{aligned}
I_{m n}^{1}(\boldsymbol{a}) & =\sum_{\beta=1}^{3} \boldsymbol{a} \cdot \hat{\boldsymbol{n}}_{\beta} \int_{\gamma_{\beta}} x^{m} y^{n} \hat{\phi} \mathrm{d} \gamma \\
& =-b \int_{0[y=0]}^{1} x^{m} y^{n} \mathrm{e}^{a x} \mathrm{~d} x-a \int_{0[x=0]}^{1} x^{m} y^{n} \mathrm{e}^{b y} \mathrm{~d} y+(a+b) \mathrm{e}^{b} \int_{0}^{1} x^{m}(1-x)^{n} \mathrm{e}^{s x} \mathrm{~d} x \\
& =-b I_{m, 0}(a) \delta_{n, 0}-a I_{n, 0}(b) \delta_{m, 0}+(a+b) \mathrm{e}^{b} I_{m, n}(s),
\end{aligned}
$$

in which $s=a-b$. The closed-form expression for the second integral can be expressed as

$$
\begin{aligned}
I_{m n}^{2}(\boldsymbol{a})= & \sum_{\beta=1}^{3} \int_{\gamma_{\beta}} \nabla\left(x^{m} y^{n}\right) \cdot \hat{\boldsymbol{n}}_{\beta} \hat{\phi} \mathrm{d} \gamma \\
= & -\int_{0}^{1} n x^{m} y^{n-1} \mathrm{e}^{a x} \mathrm{~d} x-\int_{0}^{1} m x^{m-1} y^{n} \mathrm{e}^{b y} \mathrm{~d} y \\
& +\mathrm{e}^{b} \int_{0}^{1}\left[n x^{m}(1-x)^{n-1}+m x^{m-1}(1-x)^{n}\right] \mathrm{e}^{s x} \mathrm{~d} x \\
= & -I_{m, 0}(a) \delta_{n, 1}-I_{n, 0}(b) \delta_{m, 1}+\mathrm{e}^{b}\left[n I_{m, n-1}(s)+m I_{m-1, n}(s)\right] .
\end{aligned}
$$




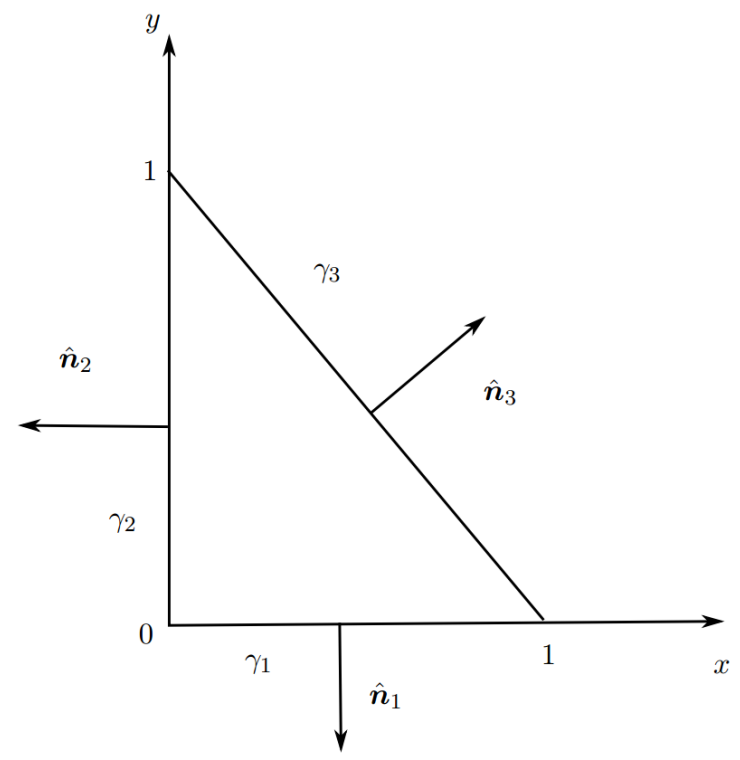

Figure 11: The integration domain of the reference triangle in the local coordinates system.

The closed-form expression of third integral can be derived based on the previous calculated integrals, which is given by

$$
I_{20}^{3}(\boldsymbol{a})=I_{02}^{3}(\boldsymbol{a})=\frac{2}{\|\boldsymbol{a}\|^{2}} I_{00}^{1}(\boldsymbol{a}) .
$$

The line integrals are all expressed in terms of the elementary integrals

$$
I_{m, n}(s)=\int_{0}^{1} x^{m}(1-x)^{n} \mathrm{e}^{s x} \mathrm{~d} x
$$

For the purposes of avoiding round off errors, enhancing the numerical stability as well as saving computational time, Evans and Webster [23, 15] have shown that these integrals can be obtained via the recurrence:

$$
\begin{gathered}
Q_{m}(s)=\int_{0}^{1} x^{m} \mathrm{e}^{s x} \mathrm{~d} x, \\
Q_{m}(s)=\left[\mathrm{e}^{s}-m Q_{m-1}(s)\right] / s,
\end{gathered}
$$

where $Q_{0}(s)=\left(\mathrm{e}^{s}-1\right) / s$ and $Q_{m}(0)=1 /(m+1)$ when $s=0$. The other integrals can be easily recovered once all monomials have been computed.

\section{References}

[1] J.M. Melenk, I. Babuška, The Partition of unity finite element method: Basic theory and applications, Comput. Meth. Appl. Mech. Eng. 139 (1996) 289-314.

[2] T. Huttunen, P. Monk, J.P. Kaipio, Computation aspects of the ultra weak variational formulation, J. Comput. Phys. 182 (2002) 27-46.

[3] E. Deckers, O. Atak, W. Desmet, The wave based method: An overview of 15 years of research, Wave Motion 51 (2014) $550-565$.

[4] C. Farhat, I. Harari, L. Franca, The discontinuous enrichment method, Comput. Meth. Appl. Mech. Eng. 190 (2001) 6455-6479.

[5] H. Riou, P. Ladevèze, B. Sourcis, The multiscale VTRC approach applied to acoustics problems, J. Comput. Acoust. 16 (2008) 487-505.

[6] L. Kovalevsky, P. Ladevèze, H. Riou, M. Bonnet, The Variational Theory of Complex Rays for three-dimensional Helmholtz problems, J. Comput. Acoust. 201250021 (2012). 
[7] O. Laghrouche, P. Bettess, Short wave modelling using special finite elements, J. Comput. Acoust. 8(1) (2000) 189-210.

[8] E. Perrey-Debain, O. Laghrouche, P. Bettess, J. Trevelyan, Plane wave basis finite elements and boundary elements for three dimensional wave scattering, Philosophical Transactions of the Royal Society of London A 362 (2004) 561-577.

[9] R.J. Astley, P. Gamallo, Special short wave elements for flow acoustics, Comput. Meth. Appl. Mech. Eng. 194 (2005) $341-353$.

[10] J.-D. Chazot, B. Nennig, E. Perrey-Debain, Performances of the Partition of Unity Finite Element Method for the twodimensional analysis of interior sound field with absorbing materials, J. Sound Vib. 332 (2013) 1918-1929.

[11] J.-D. Chazot, E. Perrey-Debain, B. Nennig, The partition of unity finite element method for the numerical solution of waves in air and poroelastic media, Journal of Acoustical Society of America 135 (2014) 724-733.

[12] R. Sugimoto, P. Bettess, J. Trevelyan, A numerical integration scheme for special quadrilateral finite elements for Helmholtz equation, Comm. Num. Meth. Eng. 19 (2002) 233-245.

[13] P. Bettess, J. Shirron, O. Laghrouche, B. Peseux, R. Sugimoto, J. Trevelyan, A numerical integration scheme for special finite elements for Helmholtz equation, Int. J. Num. Meth. Eng. 56 (2003) 531-552.

[14] W.B. Gordon, Far-field approximation to the Kirchhoff-Helmholtz representations of scattered fields, IEEE Transactions on Antennas and Propagation 23(4) (1975) 590-592.

[15] G. Gabard, Exact integration of polynomial exponential products with application to wave-based numerical methods, Comm. Num. Meth. Eng. 25 (2009) 237-246.

[16] A. El Kacimi, O. Laghrouche, Improvement of PUFEM for the numerical solution of high-frequency elastic wave scattering on unstructured triangular mesh grids, Int. J. Num. Meth. Eng. 84 (2010) 330-350.

[17] R. Hospital-Bravo, J. Sarrate, P. Díez, A semi-analytical scheme for highly oscillatory integrals over tetrahedra, Int. J. Num. Meth. Eng. (2017) DOI: 10.1002/nme.5474.

[18] M.J Peake, J. Trevelyan, G. Coates, The equal spacing of N points on a sphere with application to partition-of-unity wave diffraction problems, Eng. Ana. Bound. Ele. 40 (2014) 114-122.

[19] E. Perrey-Debain, Plane wave decomposition in the unit disc: convergence estimates and computational aspects, Journal of Computational and Applied Mathematics 193(1) (2006) 140-156.

[20] R. Duraiswami, Z. Li, D.N. Zotkin, E. Grassi, N.A. Gumerov, Plane-wave decomposition analysis for spherical microphone arrays, IEEE Workshop on Applications of Signal Processing to Audio and Acoustics, New Paltz, NY, 2005.

[21] N.A. Gumerov, R. Duraiswami, Fast multipole methods for the Helmholtz equation in three dimensions, Elsevier, (2004).

[22] P.M. Morse, K.U. Ingard, Theoretical Acoustics, Princeton University Press, (1987).

[23] G.A. Evans, J.R. Webster, A comparison of some methods for the evaluation of highly oscillatory integrals, J. Comput. Appl. Math. 112 (1999) 55-69. 